\title{
Archéopages
}

Archéopages

Archéologie et société

$38 \mid 07 / 2013$

Terres inhospitalières

\section{La « vallée secrète » à La Réunion. Un refuge extrême pour les esclaves " marrons » caractérisé pour la première fois par l'archéologie}

\section{Anne-Laure Dijoux}

\section{OpenEdition}

Journals

Édition électronique

URL : https://journals.openedition.org/archeopages/491

DOI : 10.4000/archeopages.491

ISSN : 2269-9872

Éditeur

INRAP - Institut national de recherches archéologiques préventives

Édition imprimée

Date de publication : 1 août 2014

Pagination : $20-23$

ISSN : 1622-8545

Référence électronique

Anne-Laure Dijoux, «La « vallée secrète » à La Réunion. Un refuge extrême pour les esclaves " marrons » caractérisé pour la première fois par l'archéologie », Archéopages [En ligne], 38 | 07/2013, mis en ligne le 01 juillet 2015, consulté le 21 janvier 2022. URL : http://journals.openedition.org/ archeopages/491; DOI : https://doi.org/10.4000/archeopages.491 


\section{La « vallée secrète » à La Réunion Un refuge extrême pour les esclaves «marrons » caractérisé pour la première fois par l'archéologie}

Anne-Laure DijouX Université de Paris 1 Panthéon-Sorbonne, UMR 7041 «Archéologies et Sciences de PAntiquité »

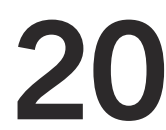

1. Effectuées en haute altitude, elles devaient relever deux défis : logistique, en assurant l'autonomie d'une équipe sur plusieurs jours, et environnemental, l'espace étant classé au cœur du Parc national et sous protection d'un arrêté de biotope.

2. Doctorat de l'auteur à l'université de Paris 1 Panthéon-Sorbonne, soutenance prévue en 2014 Recherche et étude de sites archéologiques de " marronnage » à l'île de La Réunion,

M. Gutierrez (dir.).

3. Nous remercions ces deux organismes.
Nichée à plus de $2200 \mathrm{~m}$ d'altitude au-dessus de deux anciennes caldeiras formées par le Piton des Neiges de l'île de La Réunion, la «vallée secrète» saisit l'étranger par son inaccessibilité et ses murailles rocheuses vertigineuses. Cette vallée étroite de $50 \mathrm{~m}$ et longue de $450 \mathrm{~m}$ ne se laisse aujourd'hui approcher que de deux manières : l'une, par descente en rappel depuis un pic vertigineux, l'autre, par un périlleux appui-patin en hélicoptère. Au creux de cette vallée à végétation altimontaine de fourrés et de bruyères, deux abris en pierre sèche furent aménagés contre une des parois rocheuses. Découverte fortuitement par un guide de montagne vers 1995, la zone des abris située dans le cirque de Cilaos [ill. 1] a fait l'objet de deux campagnes de sondages en 2011 et $2012^{1}$ dans le cadre d'un doctorat $^{2}$ dont les objectifs portent sur la recherche et l'étude de sites archéologiques de la période du marronnage - phénomène de fuite des esclaves dans les bois du XVII ${ }^{\mathrm{e}}$ au $\mathrm{XIX}^{\mathrm{e}}$ siècle à La Réunion, île colonisée par la France à partir de 1663 [cf. encadré p. 21]. Ces missions ont bénéficié du soutien de la direction des Affaires culturelles - océan Indien et du Parc national de La Réunion ${ }^{3}$ (Dijoux, 2012).

L'approche d'étude fut transdisciplinaire afin de mettre en évidence les stratégies humaines adoptées dans ce milieu extrême naturellement hostile à toute installation. La relative proximité chronologique entre le sujet d'étude et notre époque permettait de faire appel à des expertises botaniques. Des relevés topographiques $2 \mathrm{D}$ et $3 \mathrm{D}$ (modèle texturé) furent produits en vue de restituer et d'archiver la topographie exceptionnelle du lieu, et de visualiser les choix d'implantation et la position des végétaux d'origine anthropique.
Un refuge de haute montagne caractérisé

L'emplacement du site permet une totale dissimulation : il est impossible d'être vu à partir des deux points d'observation amont et aval, qui offrent en revanche des postes de guet à vues plongeantes et lointaines. L'emprise maximum de l'occupation reconnue s'étend sur $35 \mathrm{~m}$ de long et $10 \mathrm{~m}$ de large. La zone archéologique est implantée au centre de la vallée. Le site se compose à l'ouest, d'une plateforme présentant des vestiges de faune en surface (str. $n^{\circ} 3$ ) et à l'est de celle-ci, de deux structures aménagées en pierres sèches (str. $n^{\circ} 1$ et $n^{\circ} 2$ ) [ill. 2]. Ces deux dernières ont été adossées à un pied de falaise de sorte qu'elles soient en partie abritées par le surplomb naturel formé par la roche [ill. 3]. Elles sont orientées nord-sud par une ouverture aménagée dans leur mur sud. Le pied de falaise a servi de paroi nord à ces structures. Les pans ouest, sud et est ont été aménagés en pierres sèches provenant de blocs d'effondrement et d'éboulis disponibles dans le torrent adjacent. Des gros blocs laissés en position primaire ont été opportunément mis à profit comme angles de murs. Les caractéristiques d'un « ensemble clos » étaient donc réunies : abandon apparent, site isolé, aucune perturbation humaine ultérieure.

Les sondages réalisés dans les deux structures en pierre ont révélé la présence de foyers dans chaque abri aménagé, associés à de nombreux restes de faune majoritairement aviaire et plus ponctuellement de faune terrestre [ill. 4]. L'occupation anthropique n'est visible que sur quatre niveaux archéologiques peu épais. L'essentiel du mobilier provient de niveaux peu profonds. L'inévitable mobilité et la précarité de ces groupes humains sont attestées par la rareté du mobilier archéologique seulement composé d'un clou et d'un fragment de pipe en terre cuite. 


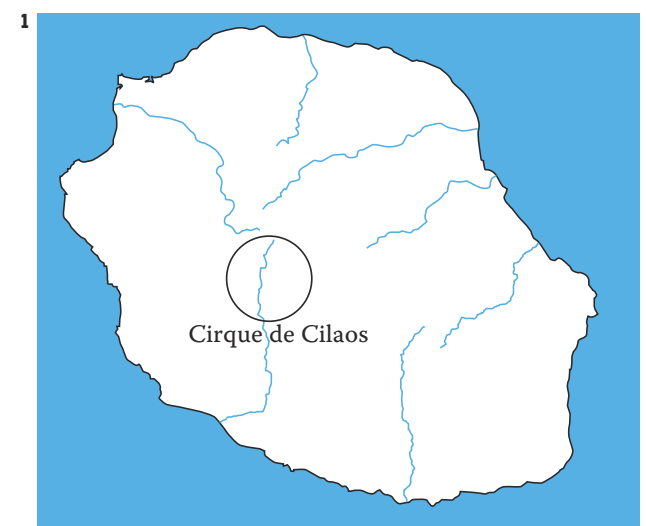

1. Carte de La Réunion,

localisation de la « vallée

secrète 1.

2. Aspect des structures

en pierres sèches, structure 1

(au fond) et structure 2

(au premier plan).

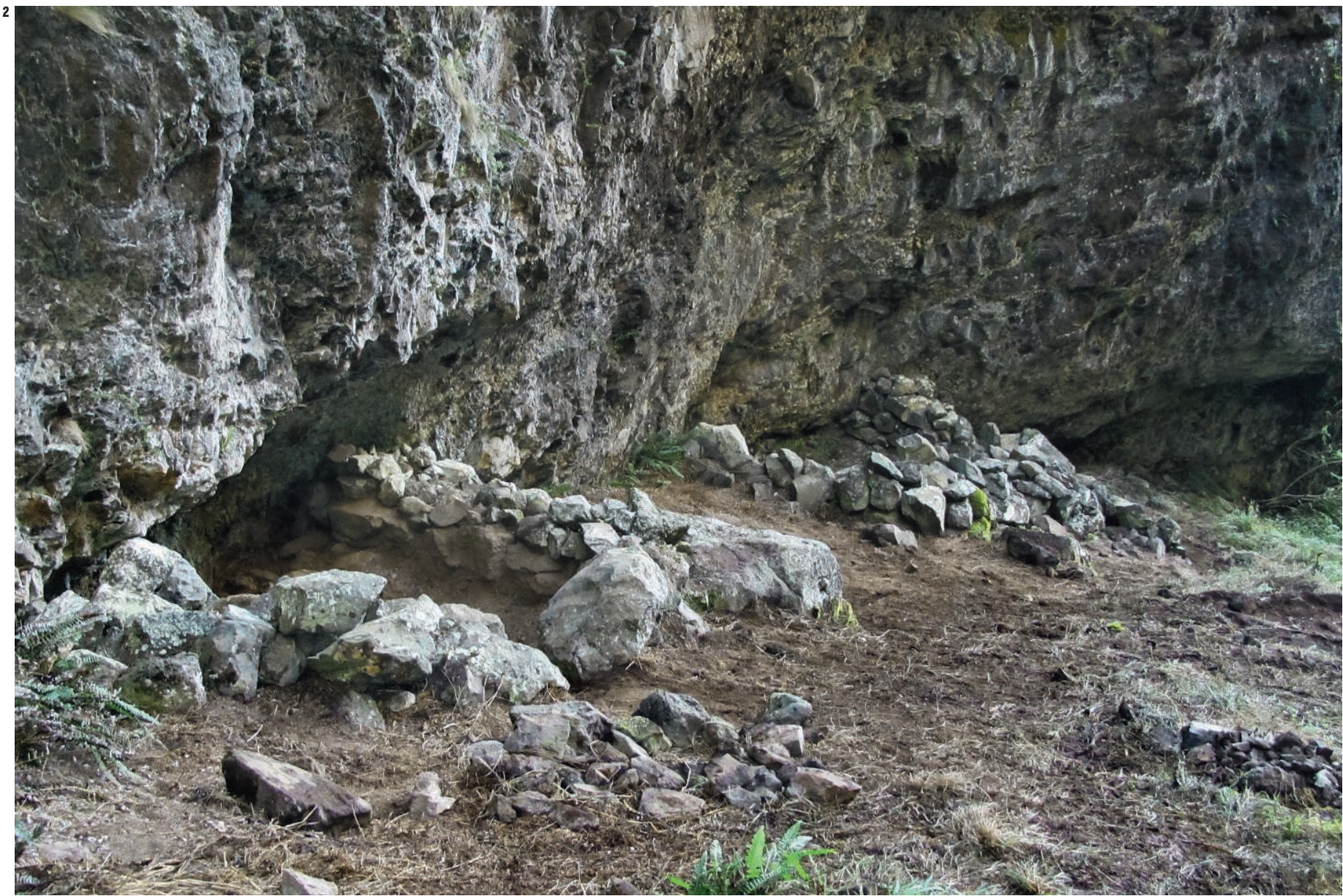

\section{Le marronnage des esclaves}

Les réflexions menées sur le site de la « vallée secrète " s'intègrent à la problématique de recherche plus large des résistances à l'esclavage colonial, qui furent nombreuses et variées. L'étude porte tout particulièrement sur le phénomène du «marronnage », fuite des esclaves dans les bois ou vers la mer, qui constitue la forme de résistance la plus directe au système esclavagiste et marque une rupture complète et volontaire de la condition d'esclave. Le " grand marronnage ", en opposition au « petit ", caractérise des fuites et des installations de longue durée, dans des lieux difficiles d'accès souvent inexplorés. Le mode de subsistance de ces groupes d'esclaves fugitifs repose principalement sur un système de chassecueillette et un nomadisme forcé. À La Réunion, tout l'intérieur de l'île, longtemps sauvage, escarpé et inaccessible, fut un terrain propice au " grand marronnage ». L'empreinte de celui-ci est aujourd'hui visible dans les toponymes d'origine malgache et africaine légués aux pitons, remparts, ravines. 
Les premières données inédites sur la matérialité du « grand marronnage »

Les observations archéologiques de la première campagne de 2011 ont démontré que la «vallée secrète» a fonctionné à la fois comme refuge imprenable et halte de chasse saisonnière où les esclaves «marrons » aménagèrent deux cabanes, se cachèrent et vinrent exploiter les ressources alimentaires disponibles en abondance à une période déterminée de l'année, dont ils avaient parfaite connaissance.

Plusieurs arguments peuvent être avancés pour appuyer l'attribution de ces structures à des esclaves fugitifs. D'abord, le caractère topographique si particulier de la vallée qui la rend très difficilement accessible et fort probablement au péril de leur vie. Ensuite, le choix d'implantation des deux structures en milieu de vallée, sous un surplomb rocheux, témoigne d'une volonté claire de dissimuler au maximum le campement. Enfin, l'extrême rareté du mobilier archéologique concorde avec la mobilité forcée de ces groupes humains nomades. La paternité de l'installation humaine à la « vallée secrète » s'oriente donc significativement vers celle d'esclaves fuyant de rudes conditions de vie dues à leur mise en esclavage dans les plantations et sucreries de l'île.

Les analyses archéozoologiques démontrent l'approvisionnement privilégié en ressources alimentaires locales par la capture d'oiseaux juvéniles ${ }^{\mathbf{4}}$ incapables de voler, directement dans leurs terriers, très abondants à proximité du site. Les quelques restes de mammifères ${ }^{\mathbf{5}}$ (porc/sanglier et chèvre/mouton), prélevés dans des zones de plus basse altitude, indiquent une grande mobilité et une pratique maîtrisée de la chasse d'animaux sauvages ou de capture de bétail. Un relevé systématique de la flore existante dans la vallée, ré-effectué récemment par un expert en botanique, révèle la présence de nombreuses espèces utiles à l'homme, autant pour leur comestibilité que pour leur caractère médicinal. Il est possible que ces espèces aient été apportées puis plantées par les esclaves fugitifs.

4. Étude de faune aviaire par Cécile MourerChauviré, université de Lyon 1.

5. Étude

archéozoologique en cours par Jean-Denis Vigne, MNHN Paris. 6. Étude des relevés botaniques en cours par Jean-Michel Probst, Parc national de La Réunion. 7. Laboratoire BETA Analytic Inc., datation réalisée en 2011, réf. 310401.

8. OSL $=$ Optically stimulated luminescence. ICP-MS = Inductively coupled plasma mass spectrometry.

9. Datation et mesures sur sédiment réalisées en 2013 par le laboratoire RE.S.Artes de Bordeaux (Dr Emmanuel Vartanian), réf. R241177A.

\section{De nouvelles données chronologiques}

La principale inconnue documentaire à déterminer sur des sites de marronnage est celle de la chronologie des occupations. En effet, de celle-ci dépend toute l'interprétation fonctionnelle des campements : refuge d'esclaves fuyant l'esclavage ou halte de braconnage plus récente, typique de la fin du XIX ${ }^{\mathrm{e}}$ siècle? Afin de répondre à cette interrogation déterminante sur le site de la "vallée secrète ", deux méthodes de datation ont été testées. En premier lieu, la traditionnelle méthode ${ }^{14} \mathrm{C}$ a été expérimentée sur un échantillon de charbon provenant du foyer de la structure $n^{\circ} 2$. Les résultats obtenus $^{7}$ (âge conventionnel : $170 \pm 30 \mathrm{BP}$ ) ont livré quatre fourchettes de datation calibrées s'étalant du XVII ${ }^{\mathrm{e}}$ au Xx ${ }^{\mathrm{e}}$ siècle, qui démontrent ainsi les limites de ce type de datation absolue appliquée à des sites d'occupation moderne et/ou contemporaine. En l'absence de données chronologiques probantes, le recours à une autre méthode de datation absolue s'est avéré nécessaire suite à la campagne de 2012. Compte tenu de la quasiabsence de mobilier archéologique et de la problématique de recherche, le fragment de pipe en terre cuite a dû faire l'objet d'une datation appropriée par OSL couplée à des mesures par ICP-MS ${ }^{\mathbf{8}}$. Cette méthode, basée sur le même principe de la thermoluminescence, prend en compte l'irradiation naturelle reçue par le sédiment et l'énergie emmagasinée par les rochers qui l'entourent. Les résultats, très fiables, révèlent une datation du dernier chauffage de la pipe en 1822 A.D. $\pm 13 \mathrm{ans}^{9}$, soit entre 1809 et 1835 . Ils nous orienteraient donc vers une occupation du site au début du $\mathrm{XIX}^{\mathrm{e}}$ siècle, soit en pleine période de l'esclavage dont l'abolition fut prononcée dans les colonies françaises en 1848. Néanmoins, il reste tout à fait plausible d'envisager une occupation plus ancienne au XVIII ${ }^{\mathrm{e}}$ siècle. Les archives écrites, qui ont démontré leurs limites sur le thème du marronnage réunionnais et ne mentionnent jamais la fréquentation de ce secteur, ne permettent pas d'apporter d'éclaircissements sur la chronologie du site. Les données étant lacunaires pour le XVIII ${ }^{\mathrm{e}}$ siècle, il est seulement admis pour le début du XIX ${ }^{\mathrm{e}}$ siècle qu'un pic de départ en marronnage fut constaté par les autorités. On recense en effet, au début de l'année 1830, 2453 esclaves portés « marrons dans les bois » (Ève, 2003, p. 169), soit près de 3,5\% de la population servile de l'île.

\section{Le premier site extrême de « grand marronnage » mis en évidence}

Les résultats archéologiques obtenus suggèrent que le site de la «vallée secrète » a été considéré et utilisé comme un point de passage offrant une position stratégique au sein d'un vaste territoire, probablement très emprunté par plusieurs groupes humains « marrons » (massif du Grand Bénare sur le territoire de l'Ouest notamment). À proximité du camp, la présence annuelle - uniquement pendant l'été austral réunionnais, de novembre à avril - d'une espèce d'oiseau, le Pétrel de Barau, seule source alimentaire disponible et facile à capturer, permet d'appuyer l'hypothèse d'une succession d'occupations saisonnières. Il est pertinent de noter que c'est précisément à cette période que les températures y sont supportables, tandis qu'elles oscillent aux alentours de $0^{\circ} \mathrm{C}$ pendant l'hiver austral. Un lieu qui, en outre, se trouve à proximité d'un haut sommet de lîle dont le nom malgache signifie « très grand froid ».

Située dans un milieu extrême, hostile et supposé inhabitable, la « vallée secrète » constitue le premier site fréquenté par des esclaves « marrons » caractérisé grâce à l'archéologie. Bien que pionniers et très informatifs, ces résultats concernent uniquement un site, par ailleurs exceptionnel (Dijoux, à paraître). D’autres données seront nécessaires afin de déterminer tous les types de stratégies d'adaptation et de modes d'occupation employés par les groupes d'esclaves fugitifs dans les terres intérieures de l'île de La Réunion. 


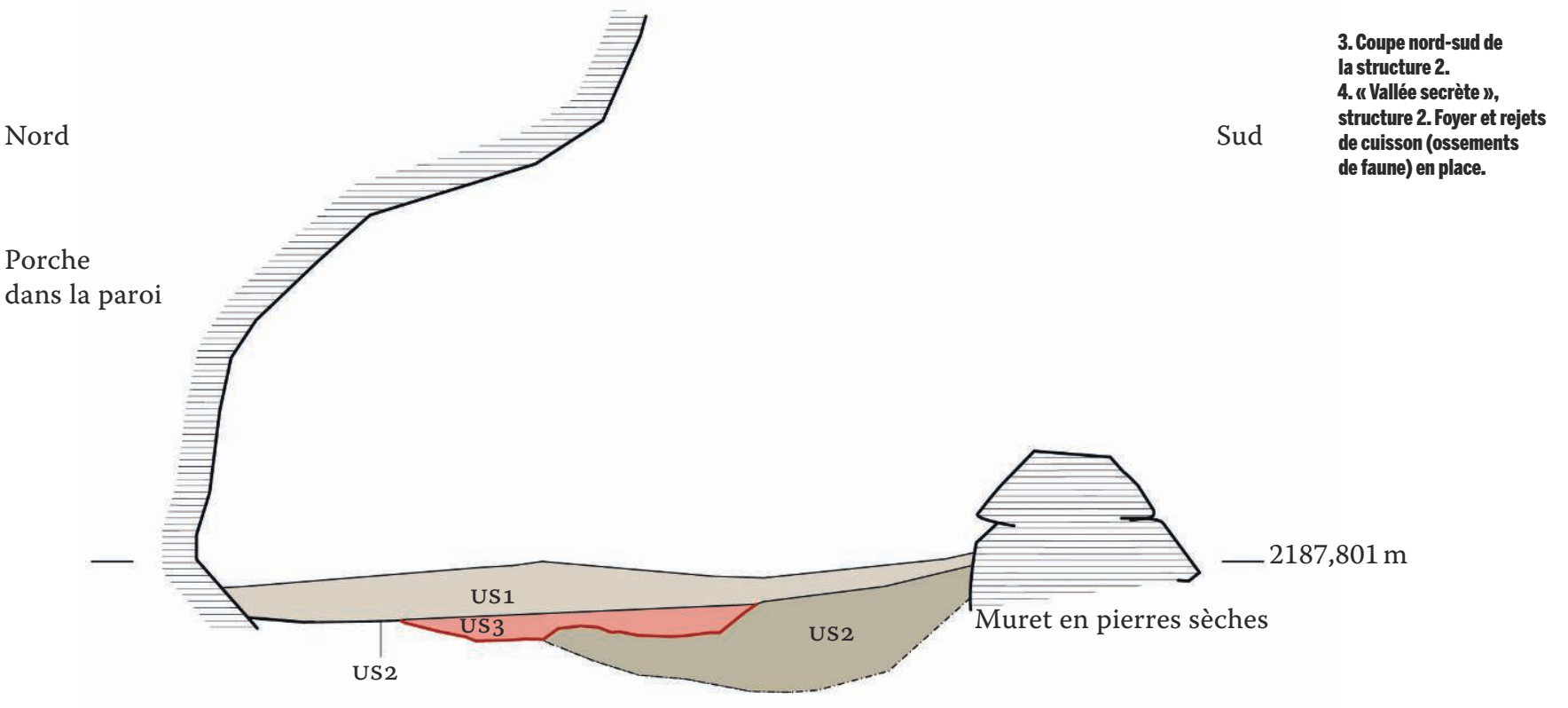

Nord

Porche

dans la paroi

Paroi rocheuse

4

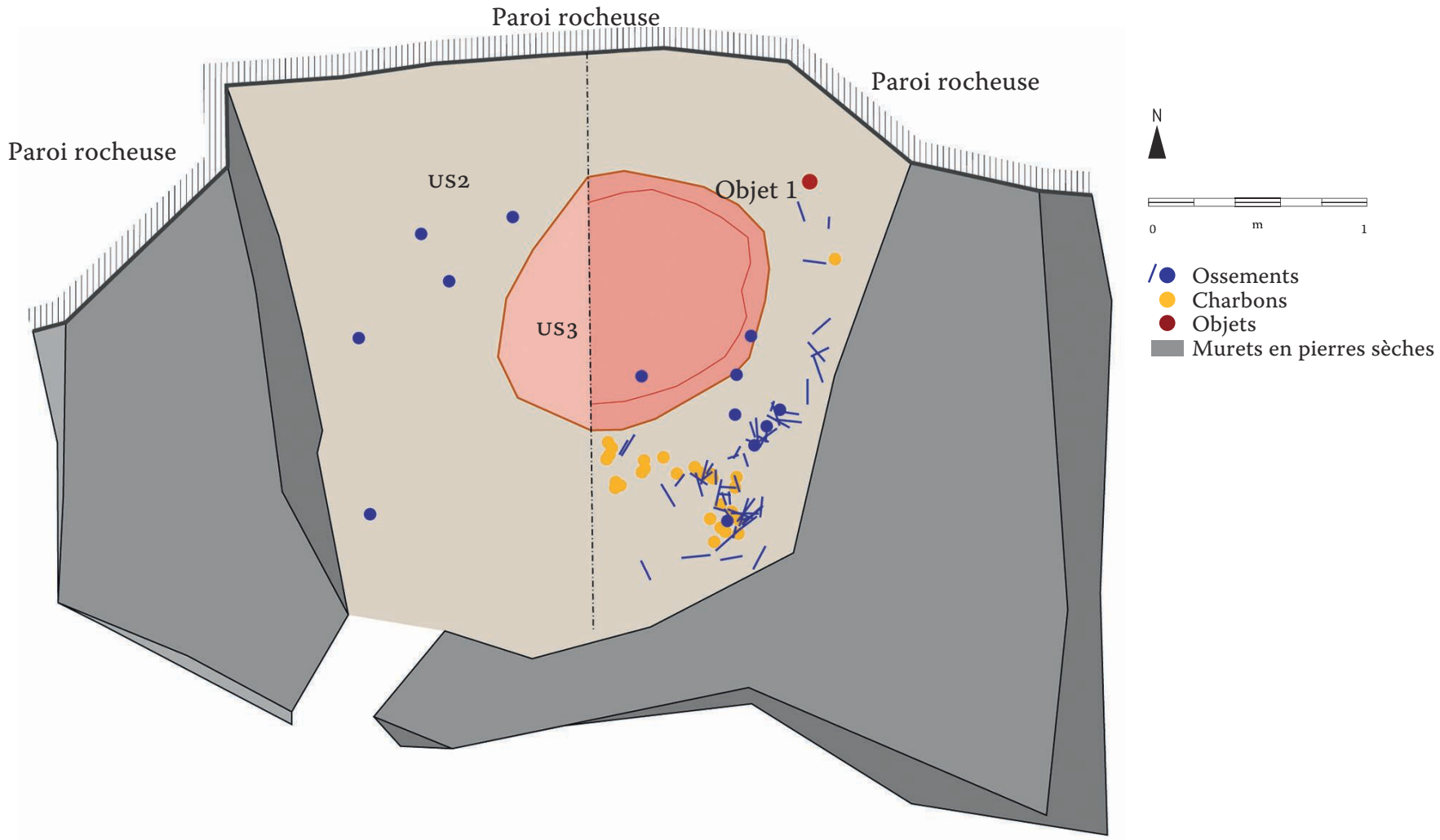

Références bibliographiques

Dijoux A.-L., à paraître : « L'archéologie du marronnage à lîle de La Réunion : l'exemple du site de la "vallée secrète" dans le cirque de Cilaos », in Actes du colloque "Archéologie de l'esclavage colonial», Musée du quai Branly, 9-12 mai 2012, Paris, Inrap. Dijoux A.-L., 2012 : Opération archéologique programmée sur les sites de la "vallée secrète » et du "Piton Béthoune » en juillet 2011 dans le cirque de Cilaos (97 424), La Réunion, Rapport intermédiaire de prospections et de sondages archéologiques, 112 p. (rapport non publié, communication soumise à autorisation du service de l'archéologie de la Dac-oI).

Ève P., 2003: Les esclaves de Bourbon, la mer et la montagne, Paris - Saint-Denis de La Réunion, Karthala - Université de La Réunion. 